\title{
Measuring system for parallel moving ships
}

\author{
Anna Waszkiel, M.Sc., \\ Gdynia Maritime University
}

\begin{abstract}
The paper introduces algorithm for determining the relative positions of two ships manoeuvring as a pair. This algorithm also takes into account determination of angle $\gamma$, which is difference between present approaching vessel and guidance vessel course. Relative positioning system is a vision system based on three colours LEDs matrix and rotating $C C D$ camera. There are presented ways of distance calculation based on photogrammetric methods from the known distance between the characteristic points of the real. Several possible cases are taken into account. The considerations have been illustrated on the basis of model of the system. These results confirm the correctness of the operation of the algorithm that is used by the designed measuring system.
\end{abstract}

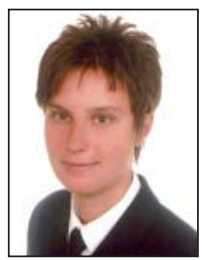

Keywords: vision system; relative ship position determination; safe ship control; computer simulation

\section{INTRODUCTION}

Each ship manoeuvring on the marine area, for safety navigation, should have information about courses and position of the other vessels navigating in the vicinity. Safety of units being in motion is subjected to the consideration of the hydro-meteorological factors, navigational dangers (eg. shallow waters, rocks, wrecks) and other vessels positions in multidimensional steering process. In ships control system information form electro navigational systems like GPS, AIS, radar and ship's log is used for this purpose. This information can be derived also by navigator's decision support system, like ARPA. Taking into account two vessels moving in parallel, safety of the manoeuver depends not only on proper ships location in navigational space, but also on accurate relative position determination. Underway Replenishment Operations (UNREP), fishing boats trawling in pair, pipe laying operations and some research situations are real examples of parallel ships movement on the sea.

For the safe performance of manoeuver in vessels parallel motion, it is necessary to pinpoint transversal and longitudinal distance between ships and also to determine parallelism of the course. In case of no parallelism of ship's courses it is necessary to determine angle $\gamma$, which is difference between present approaching vessel and guide ships course, which is shown in the Fig. 1.

When taking into account manoeuver of ships parallel movement, whole procedure consisting of three phases should be taken into consideration. It is not only case when vessels are arranged side to side amidships. This exemplary operation is divided into three phases: approaching, parallel movement and departure, which is shown in the Fig. 2.

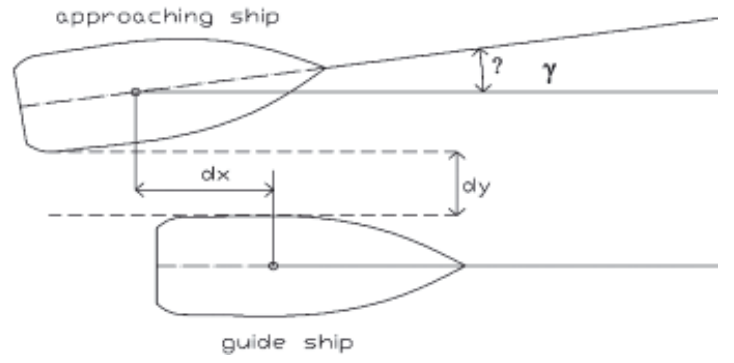

Fig. 1. Approaching and guide ships relative position coordinates

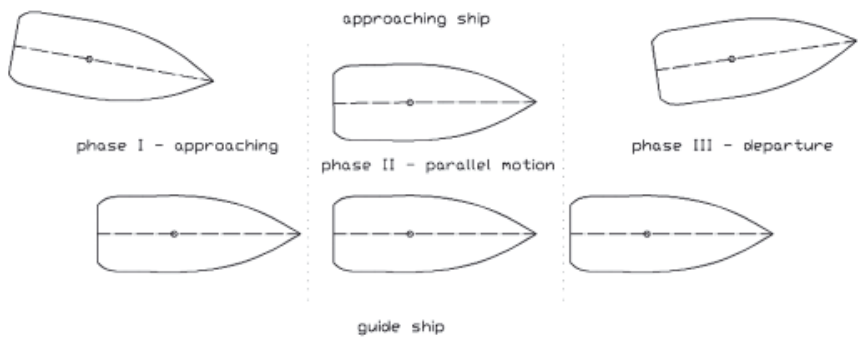

Fig. 2. Phases of the pair of the whole ships manoeuver containing parallel motion

Approaching and departure are the most dangerous phases, because of big influence of forces and yawing moments acting on vessels bodies. Parallel movement is easier to control, because only suction force is significant, there is hardly any yaw moment there due to suction phenomenon [7]. So it is important to develop relative position counting algorithm, which will work properly not only in parallel movement, but also in first and third phase. 


\section{METHODS FOR DETERMINING RELATIVE POSITIONS OF THE SHIPS}

In case of two ships manoeuvring on the sea surface only contactless measurement methods can be applied. Distance can be measured directly or computed analytically from shape measurement on the basis of mathematical transformations. This is possible using sound waves (ultrasonic rangefinders) [10] or light waves $[2,5]$ (optical methods) as a medium. Relative position and course determination in marine navigation systems is based on radar data. Radar angular discrimination should be less than $1^{0}$ and distance discrimination should reach $20 \mathrm{~m}$ or $1.5 \%$ of range according to IMO Performance Standards [6]. Taking radar into account there is also problem of shadow areas and reflections from water surface in the vicinity of the ship. Discrimination values for ships models are to big to rely on them in relative position estimation system. In addition radar in its working principle uses microwave radiation, which is unhealthy for the crew working in a small distance from the other ship. Radar could be treated as additional element of the whole positioning system during approaching and departure phase.

Optical measurement methods are based on measuring the reflection or scattering on the surface of the test object. The most common method of the optical measurement is based on the laser triangulation. Triangulation phenomenon was first time mathematicaly described by W. Snell van Royen in 1615. Over the past 20 years there has been seen development of this method [5], caused by CCD cameras widespread usage and laser technology development. The next well known optical distance measurement method is structured light projection. The method is based on raster patterns projection onto the surface of an object. Then observations carried out with a camera system are analysed. Received patterns are processed and on the basis of analytical dependences the distance is obtained [4].

For a mutual vessel's position evaluation system it is not possible to apply any of the presented above methods. Coneshaped bow makes laser range-finders distance measurements unusable. There is no simple way to verify to the which side of the vessel the measurement was made, when the $\gamma$ angle is negative and/or big (Fig. 1). Furthermore computer analysis would require knowing the exact shape of the hull. This would make the system possible to use only on board of certain type of ships. Structural light projection is a method which for physical reasons - like strong solar light, dark scratched hull, could not give desired effects. Of these reasons decision was made, to use photogrammetric methods to assess the relative positions of two vessels.

Photogrammetric measurements, whose precursor was the Frenchman A. Laussedat use the principle of the human eye. They use the physical phenomena of perspective and parallax. They are based on picture or the individual video frames analysis. Photograph (or video frame) is a perspective (middle) view of an object on a matrix plane [3], as shown in Fig. 3.

$$
\frac{D}{D_{x}}=\frac{f}{d_{f}} \Rightarrow D=\frac{D_{x} f}{d_{f}}
$$

On the basis of the known pixel dimensions, camera focal length (f) and the real distance between characteristic points $\left(D_{x}\right)$ it is possible to calculate the distance between the recording system and the object according to (1). These characteristic points in this vision system are blue and red light sources.

The main aim of the research carried out is to develop an algorithm which allows to assess the relative positions of the two ships manoeuvring in the vicinity treated as a pair, using photogrammetry measurements and basing on structurally simple measuring system. The research was carried out on the basis of the film realized with the use of construction in which LED's were placed. Construction model was made in 1:2 scale. The algorithm has been developed in Matlab environment, where also were carried out simulation studies.
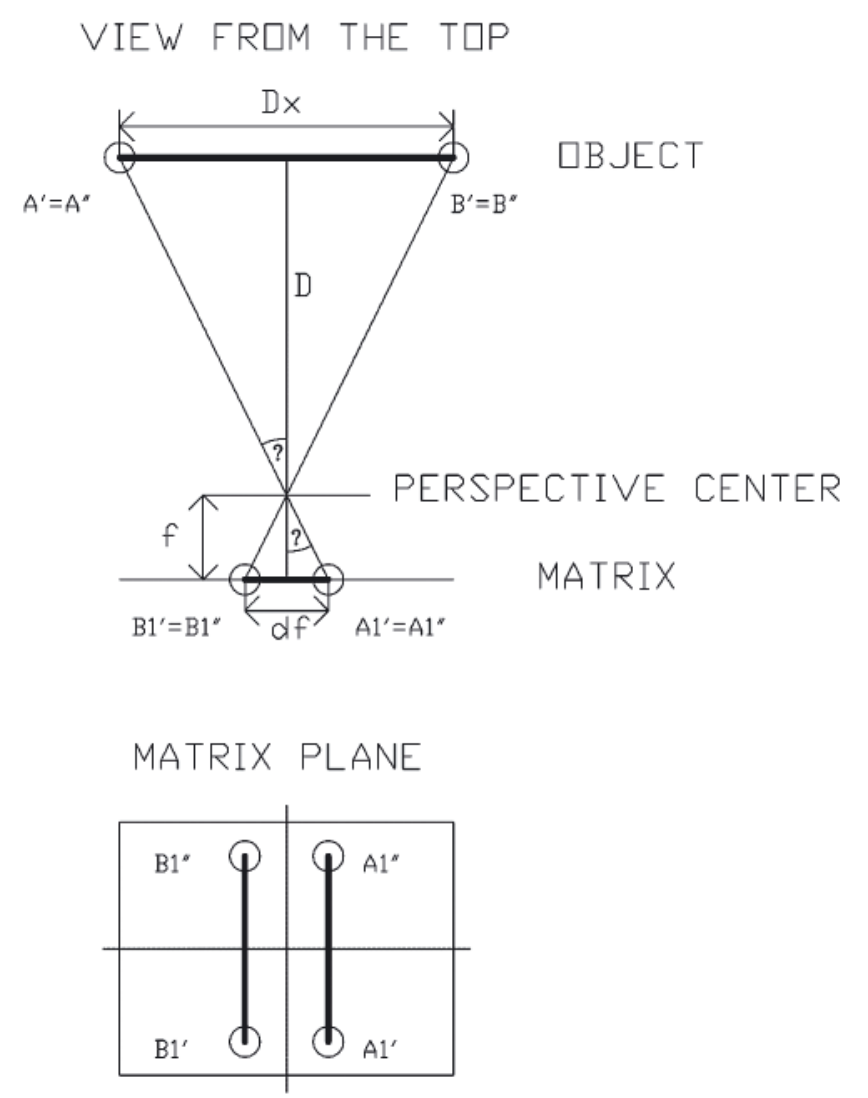

Fig. 3. The central perspective projection - representation of the actual image on the camera sensor

\section{MEASUREMENT DEVICES - SYSTEM DESCRIPTION}

For carrying out the tests was built measurement system consisting of measurement board, rail, camera for image acquisition, servo mechanism, laser rangefinder, xPC Target (Real-Time System) and notebook. This is shown in the Fig. 4.

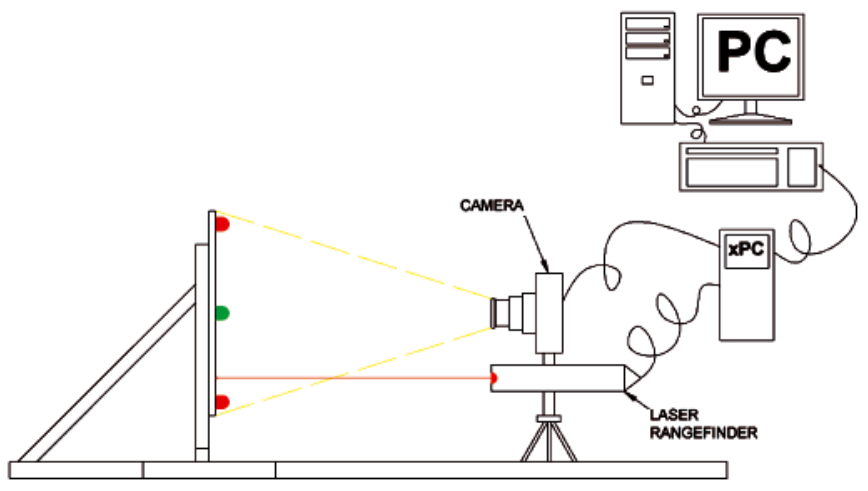

Fig. 4. The measurement system

Measurement board is the place where five light points created by LEDs are placed. There are two blue and two red points and one green point in the middle, as shown in the Figure 5. The board is placed on a trolley on the rail, to allow changing the distance between the optical system and 
the points of light. As a recording system was used Samsung S1070 camera with 10MPix CCD image sensor [9]. Its real size is $1 / 2.33$ ", which in terms of SI units gives the dimensions: $6.13 \times 4.60 \mathrm{~mm}$ and the diagonal length is $28 \mathrm{~mm}$. The research was carried out with a fixed focal length $f=6.3 \mathrm{~mm}$ of the optical system. Video material was recorded with a resolution of $640 \times 480$ [pix]. In order to allow analytical calculations of the distance between measurement board and camera image sensor, the pixel size $\mathrm{p}$ in $\mathrm{mm}$ was determined by the formula (2). Pixel size estimated from the available technical data of the camera [8] is $9.6 \mu \mathrm{m}$.

$$
\mathrm{p}=\frac{\mathrm{a}}{\mathrm{r}_{\mathrm{a}}}=\frac{\mathrm{b}}{\mathrm{r}_{\mathrm{b}}}
$$

where:

$\mathrm{p}-$ pixel size in $[\mathrm{mm}]$,

a - length of the longer side of the matrix [mm],

b - length of the shorter side of the matrix [mm],

$r_{a}-$ number of pixels per longer side of the matrix,

$r_{b}-$ number of pixels per shorter side of the matrix.

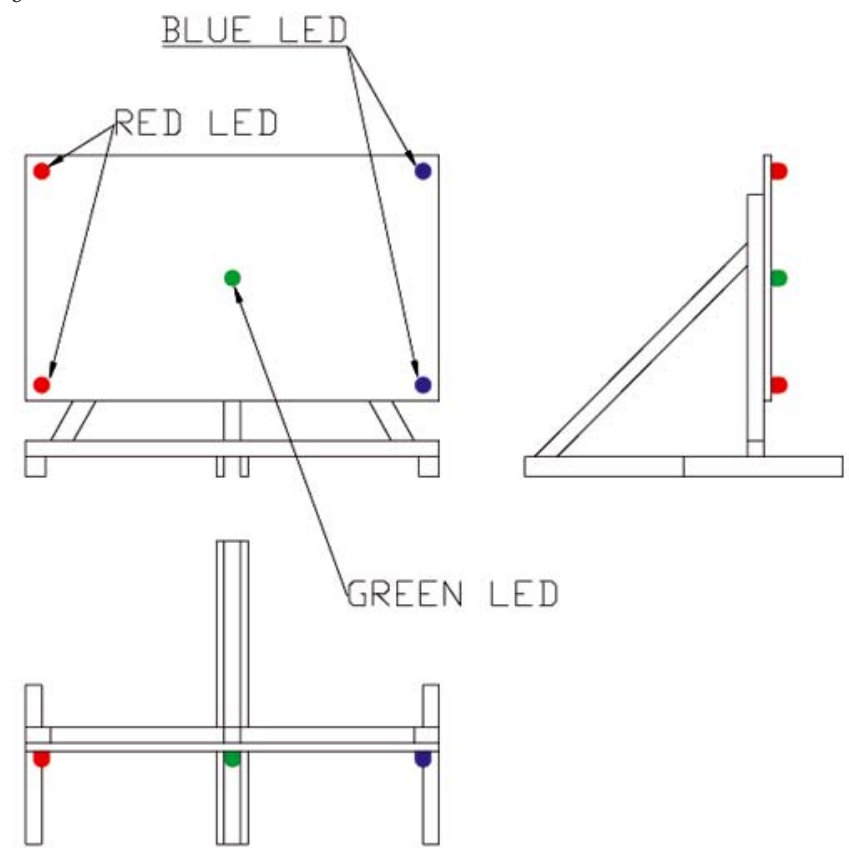

Fig. 5. The measurement board with blue, red and green LED points

The distance from camera to the measurement board was changed by the board with LEDs at small speed movement. Camera was in a fixed location. To enable analysis of the image located at various angles to the optical axis of the camera - recording system was placed on the rotary servo. Its rotation angle was controlled by the usage of Real-Time System xPC Target. Into the measurement system came also laser rangefinder, that due to high accuracy of $10^{-3} \mathrm{~mm}$ was treated as a distance pattern. Data from laser rangefinder was recorded by real-time system. Program which controlled angle between optical axis and measurement board was written in Matlab.

\section{LED RECOGNITION ALGORITHM FOR VIDEO IMAGE}

Photogrammetry is a method for making distance calculations on the basis of particularly points which are easily distinguishable on the digital image. In the system of determining the relative positions of two vessels the board with five LED light shown in Figure 5 was used. Light points due to the much higher than the average brightness of the image are easily recognizable. The use of light sources of different colors allows for easy correlation of points on the image with the points in reality.

The detection of individual points of light is illustrated in the diagram shown in Fig. 6.

The video frame is read, and after that its color space is changed from RGB to $\mathrm{YCrCb}$. Through this operation, it is possible to analyze in each channel separately, where $\mathrm{Y}$ - means the total brightness of all pixels in the image, $\mathrm{Cr}$ - red difference and $\mathrm{Cb}$ - blue difference. Color space transformation from RGB to $\mathrm{YCrCb}$ is given by the formula (3) [1].

$$
\left[\begin{array}{c}
\mathrm{Y} \\
\mathrm{Cr} \\
\mathrm{Cb}
\end{array}\right]=\left[\begin{array}{ccc}
0.299 & 0.587 & 0.114 \\
-0.169 & -0.331 & 0.5 \\
0.5 & -0.419 & -0.091
\end{array}\right]\left[\begin{array}{l}
\mathrm{R} \\
\mathrm{G} \\
\mathrm{B}
\end{array}\right]
$$

The next step is analysis of the image brightness combined with the brightest regions selection. Having examined dozens of images of LEDs, which were done form several distances the distribution of brightness values in $\mathrm{Cr}$ and $\mathrm{Cb}$ channels for blue, green and red LED was verified. On this basis, the mean values were established, which became the thresholds in the algorithm. By comparing the brightness values around the areas selected as the brightest - is assessed whether the bright area of the picture is LED. It is also known that the middle points of red and blue LEDs (set one above another on the board) can not be apart in X direction. If in the picture was found pair of desired color diodes - the distance between them is being counted. And if not - the next video frame is analyzed. The data obtained in the form of LEDs coordinates and the distance between pairs of mono-color LEDs, are passed to the algorithm for determining the angle and distance. It gives an opportunity to determine the distance and angle between the plane in which are light diodes and the plane of image sensor. If the pair of characteristic points in the image are not found, the algorithm leading analytical calculations is not activated.

This approach helps to eliminate glare, which in the case of sunlight are white and in the case of colored light (corresponding to the colors of LEDs) - do not fulfill the condition of mutual position.

\section{ALGORITHM DISTANCE AND ANGLE BETWEEN LIGHT PLANE AND IMAGE SENSOR PLANE FIXING}

Algorithm for distance and angle works on two levels. Angle and distance between sensor matrix plane and board plane are determined separately on the basis of known real distances between LED diodes and corresponding distances on the image sensor. In connection with the construction of light layout and the occurrence of perspective phenomenon, it is known that the length of vertical segments between red and blue LEDs are equal only if both planes are parallel. In that case the angle between optical axe of camera and light board is equal to $90^{\circ}$, which is illustrated in Fig. 3. Analytical calculations of distance are not complicated and are carried out according to formula (1). There is no need for computing the angle. Described case is the simplest one and occurs only in the second phase of manoeuver, when two vessels are moving parallel and there is no longitudinal shift between them. This part of the algorithm enables quick determination of lateral shift, which ensures easy and quick identification of the distance between the two ships. In the remaining stages of the manoeuver, apart the distance is also determined the angle between the optical axis of camera and the array of lights. In the algorithm are considered four separate cases (see Fig. 7): 


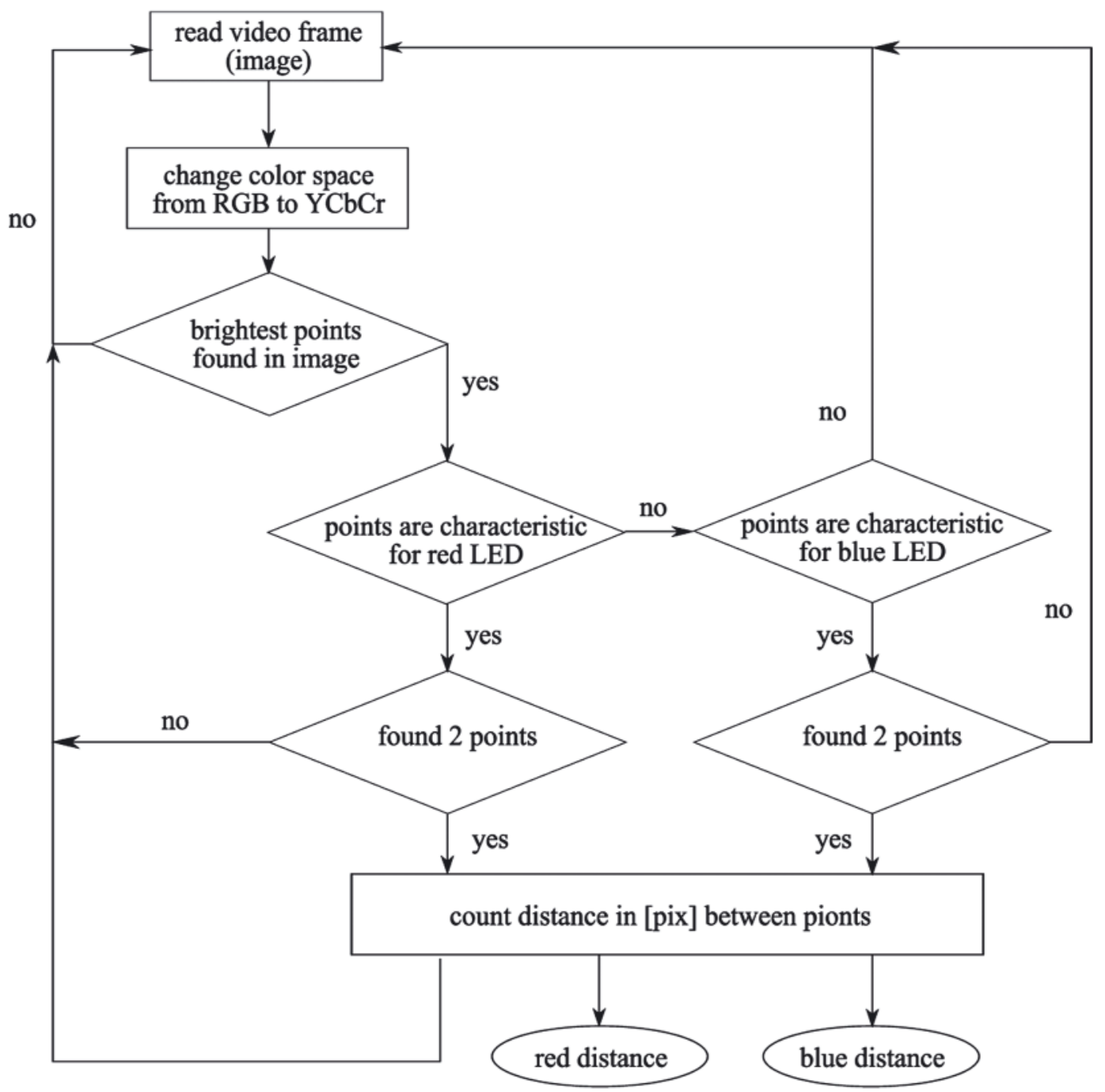

Fig. 6. LED in image recognition algorithm

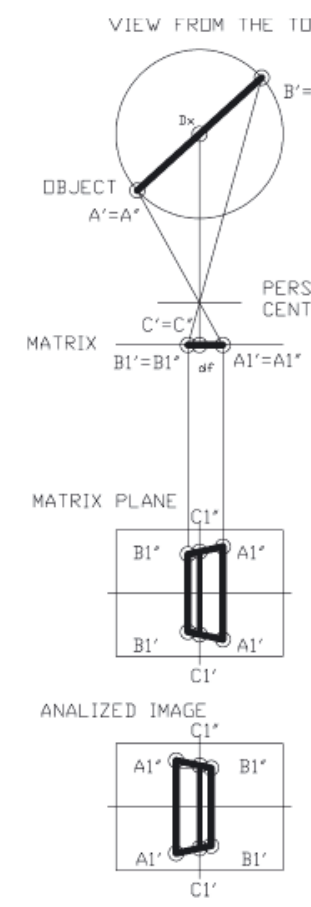

a)
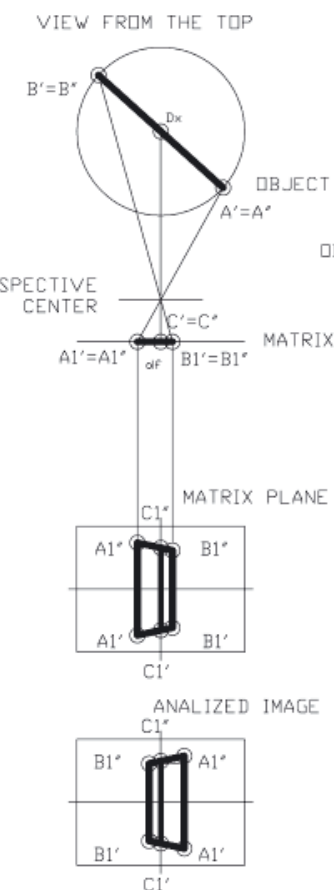

b)
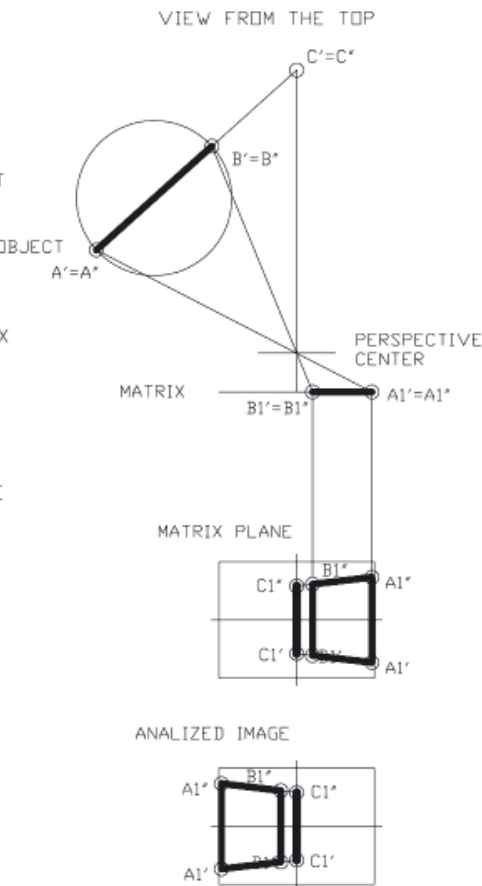

c)

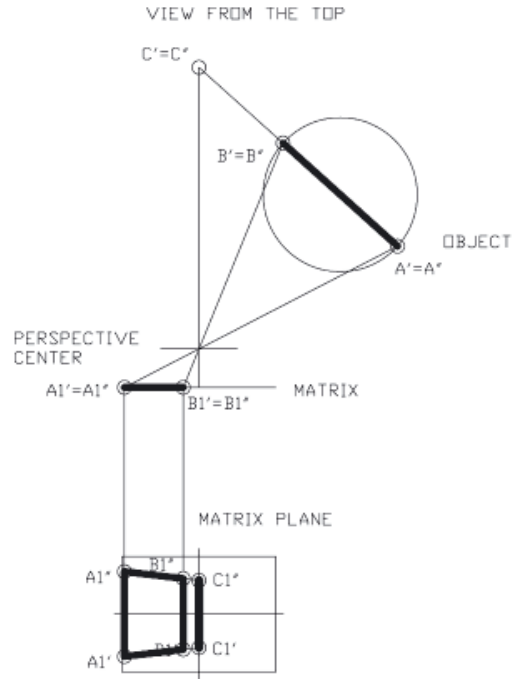

ANALIZED IMAGE

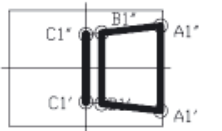

d)

Fig. 7. a) Mutual LEDs locations on the video image and in reality - case a; b) Mutual LEDs locations on the video image and in reality - case b; c) Mutual LEDs locations on the video image and in reality - case c; d) Mutual LEDs locations on the video image and in reality - case d 
a. LEDs are located on both sides of the matrix center and segment between the and the red LEDs is longer than the segment between the blue LEDs;

b. LEDs are located on both sides of the matrix center and segment between the and the blue LEDs is longer than the segment between the red LEDs;

c. all points of light are located on the left side of the matrix center;

d. all points of light are located on the right side of the matrix center,

(respectively a, b, c, d).

The basic dependence used by the algorithm is the occurrence of perspective visible in the pictures. The closer to the optical system is a segment, that it appears to have a greater length. In reality the distance between light points does not change. The same rule is used during $3 \mathrm{D}$ object from photo modeling [11]. Therefore, in order to determine the distance between the point where the light is placed (eg. a ship's bow) and the point at which the optical system is set (such as stern of the second ship), the intersection points of the image should be found. The intersection points are determined by crossings of lines contain ing respectively points A1', B1' and A1", B1" (Fig. 7).

Then is determined the length of the segment $\mathrm{c}=\overline{\mathrm{Cl}^{\prime} \mathrm{Cl}^{\prime}}$, which corresponds to the distance between these lines designated in the middle of the image sensor. On this base according to mathematical relationship (1) distance $F$ is computed. This is distance between camera lens and virtual light table rotation center $\mathrm{C}$. Distance marked as $\mathrm{F}_{\mathrm{x} 2}$ in the Fig. 8 is the longitudinal distance, which will be passed to the control system.

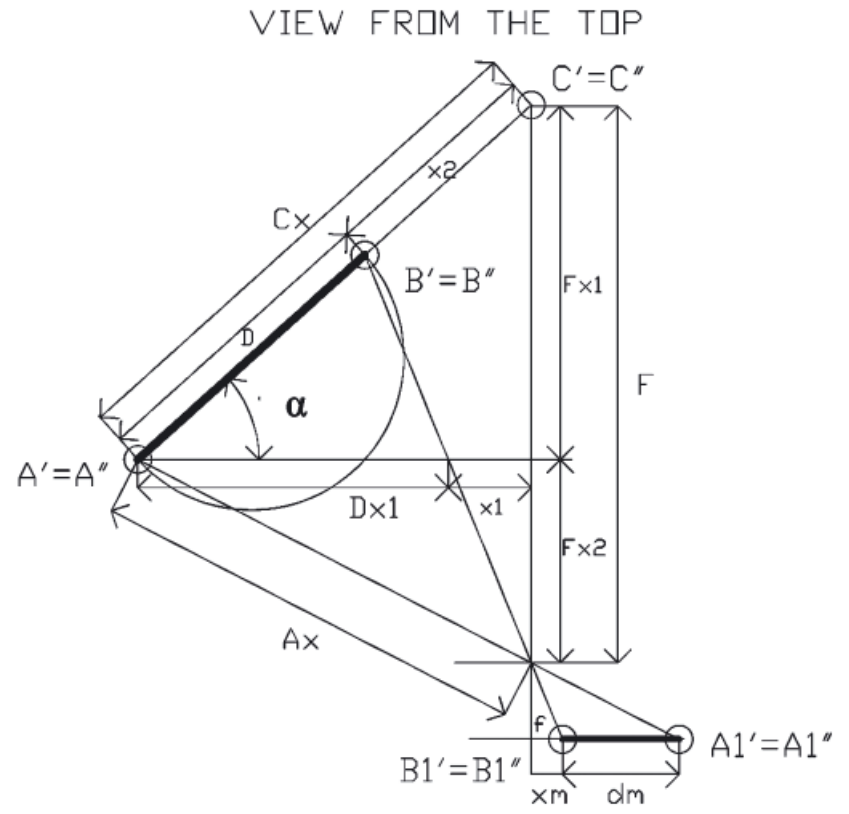

Fig. 8. Central perspective projection - determination of the angle and distance.

In order to determine the angle between the plane of the measurement system, and the plane of the image sensor matrix are the basic assumptions in analytical geometry were used [eq. (4), (5), (6), (7)]. Angle $\alpha$ is being counted in each algorithm step on the basis of equation (8), where the corresponding distances are marked in the Fig. 8.

$$
A_{x}=\frac{D_{y} f}{d_{m} p}
$$

$$
\begin{gathered}
F_{x 1}=\frac{A_{x} f}{\sqrt{f^{2}+\left(d_{m} p+x_{m}\right)^{2}}} \\
C_{x}=\sqrt{\left(F-F_{x 1}\right)^{2}+\left(\frac{F_{x 1}\left(d_{m}+x_{m}\right)}{f}\right)^{2}} \\
F_{x 2}=F-F_{x 1} \\
\alpha=\arcsin \left(\frac{F_{x 2}}{C_{x}}\right)
\end{gathered}
$$

where:

$\mathrm{D}_{\mathrm{y}}$ - the real distance between two red and two blue LEDs,

f - the focal length of the camera lens,

$\mathrm{p} \quad-$ pixel dimension $[\mathrm{mm}]$.

Distance is being counted in $\mathrm{mm}$ and angle $\alpha$ in degrees. Algorithm recalculates the angle value into system values $0^{\circ} \div 180^{\circ}$, where $0^{\circ}$ is parallel movement and $180^{\circ}-$ means that ship is moving parallel but on the other side. The second case is almost impossible to obtain in practice.

\section{RESULTS}

Research was carried out on the basis of the real model of the system made on a scale 1:2 (Fig. 4). As the first distance assessment algorithm was tested. Calculations, under which was designated the distance between the camera and measurement table, were made based on the video. It was filmed at the time when the light board was moved away parallel to the optical axis of the camera along the rail.

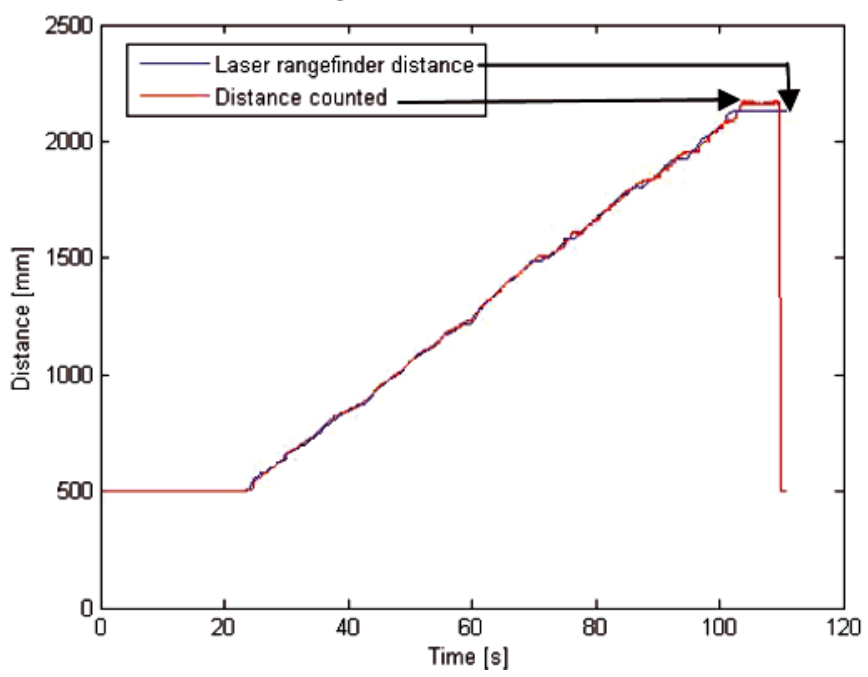

Fig. 9. Distance count and laser rangefinder measurement.

During the measurement the distance between the optical system and the table was recorded using a laser rangefinder. The results are presented in Fig. 9.

Line described by "laser rangefinder distance" and pointed by arrow indicates the reference distance carried out using laser rangefinder. The results of computations done in Matlab environment using distance evaluation algorithm are also presented in Fig. 9 and described "distance counted". The biggest difference between reference and computed distance occurs at the end of simulation and reaches $30 \mathrm{~mm}$ at range of $2.13 \mathrm{~m}$. It is about $1.55 \%$. At the rest of simulation this difference is less than $1 \%$. 
In the second stage of the study measurements, which allowed for evaluation of the correctness of the algorithm is the simultaneous assessment the angle and distance, were performed. For this purpose the rangefinder combined with a camera was placed on the rotary servo. To check if the characteristic of servo's rotation angle is linear, the calibration measurement was made. Rotating rangefinder system was placed at a fixed distance from the large size vertical calibration board. There was performed measurement of angles from 50 to 130 degrees. Servo rotation angle was set based on the basic trigonometric and registered according to laser distance measurement, which is described by "laser count" in the Fig. 10.

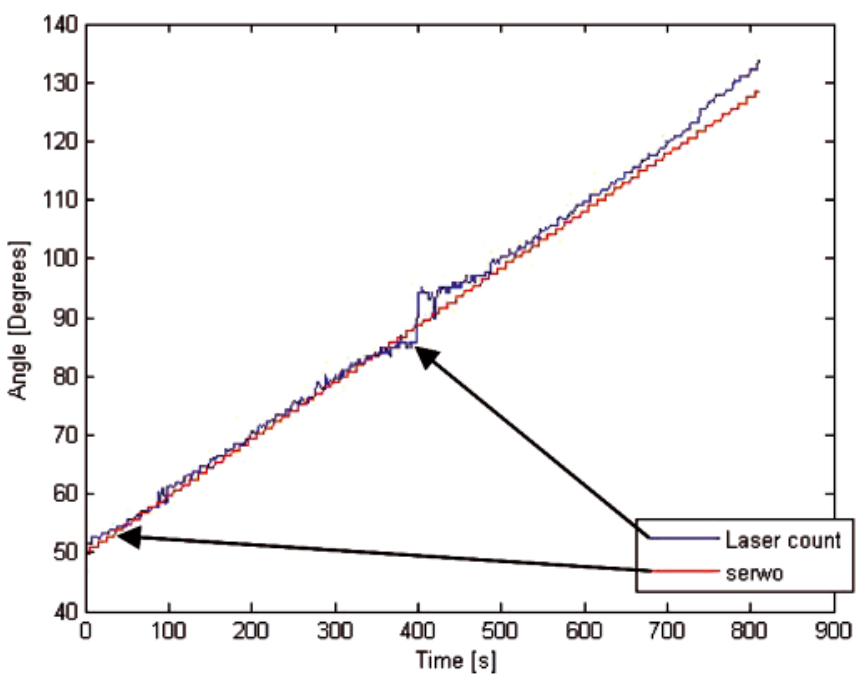

Fig. 10. Linearity of the servo rotation characteristics check

Servo rotation characteristic is close to linear one and for the algorithm will be so treated. It could be described by the following equation of straight line: $f(x)=0.97 x-55.93$.

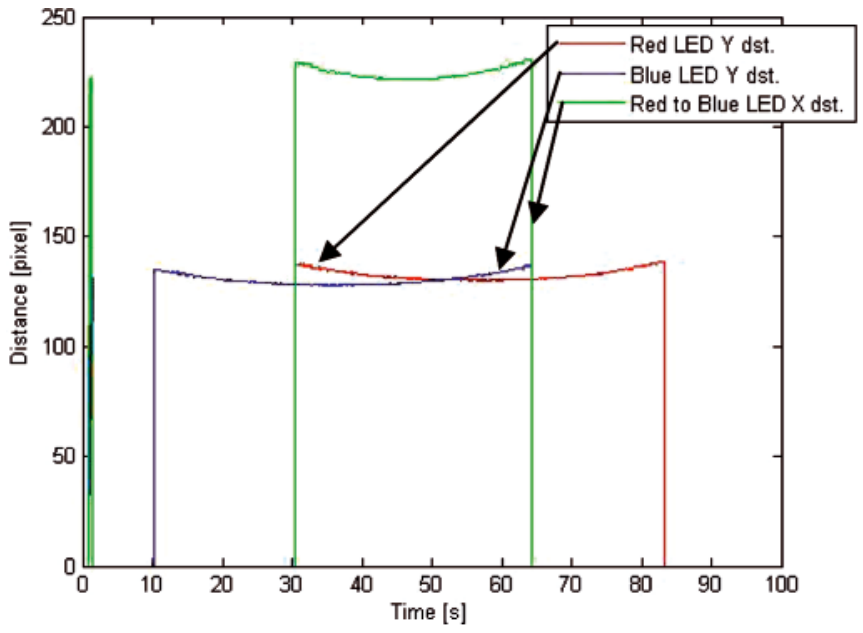

Fig. 11. Distance between LEDs estimation

Array was placed at a fixed distance of $1.52 \mathrm{~m}$ from the optical system. The camera that is treated as a video recorder was positioned on the rotary servo motor. Research was carried out on the basis of the recorded video in postprocessing mode. The control signal angle of the servo and data from measuring the distance both recorded in real time have been adopted as a benchmark. They are described by "servo angle" and "laser rangefinder" on the charts presented in Figs 12 and 13.

The distance between the centers of diodes with the same color were calculated by the algorithm and plotted in the Figure 11 marked with "Ydst". Similarly is calculated the distance between the centers of diodes located in one horizontal line. It is marked with "Xdst" in the Figure 11. Based on these distances are calculated angles and distances in the subsequent stages of the algorithm.

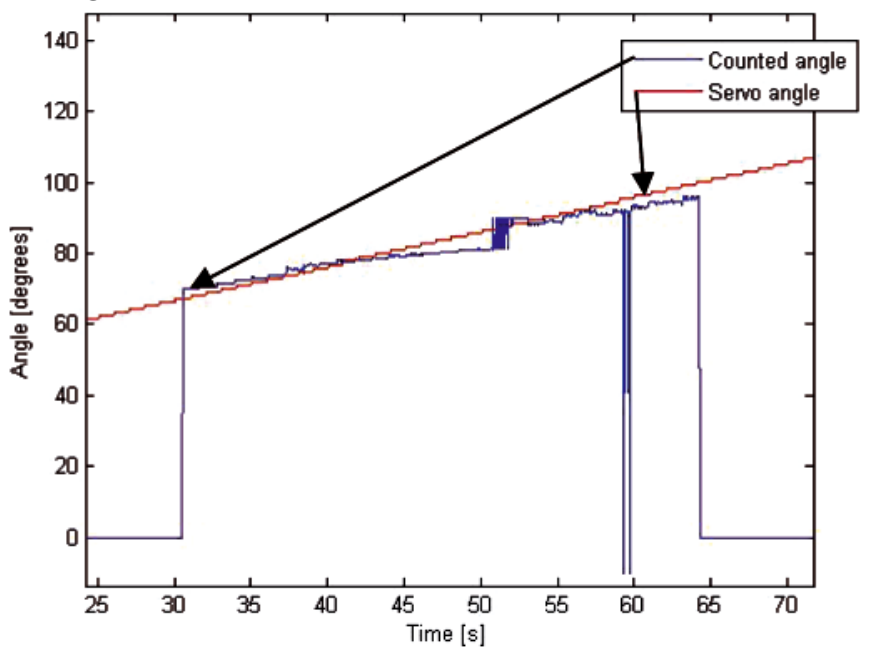

Fig. 12. Angle between the plane of light emitting diodes and the camera sensor

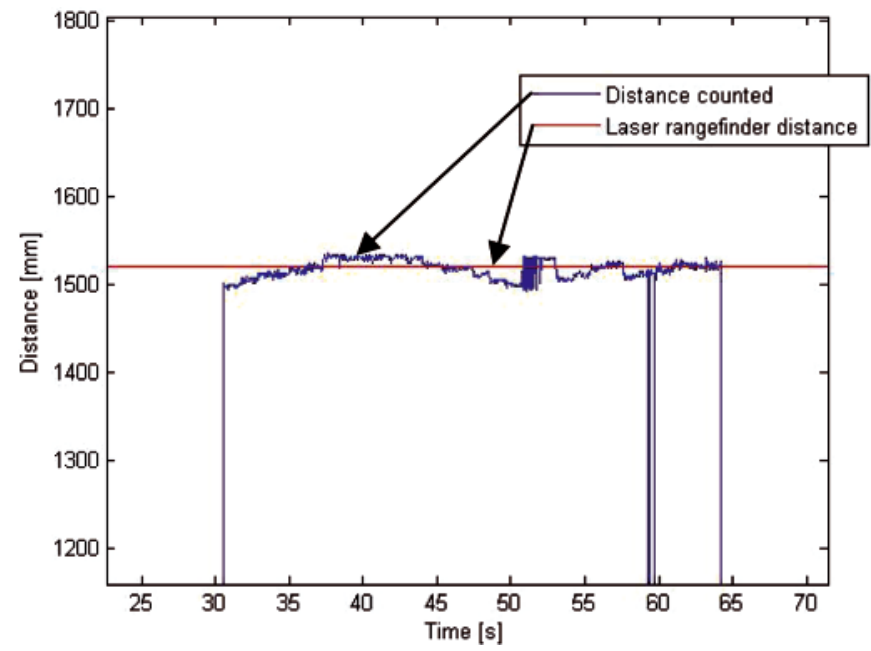

Fig. 13. Distance between the plane of light emitting diodes and the camera sensor

Angle and distance estimations are shown in the graphical form in Figs 12 and 13. The biggest difference in between reference and counted distance is about $21 \mathrm{~mm}$. Which gives $1.38 \%$ of whole distance. These differences are especially noticeable at small angles between the planes and when they are nearly parallel. The first mentioned case is probably due to defects in optics. In the second one occurs difficulty in algorithm cases switching. Also the angle difference, which reaches $5^{\circ}$ at angle of $88^{\circ}$, could be caused by the same process. It gives value at the border of acceptability about $5 \%$. At time point of 60 s distance count of $0 \mathrm{~mm}$ and angle count of 0 deg occurs. There is shown case where 4 characteristic light points were not distinguished in the analysed image. In real time system in this case samples are being filtered and the distance and angle are predicted on the basis of the previous counts.

\section{CONCLUSIONS}

- Presented algorithm can be successfully used in the control system of the relative positions of two vessels moving parallel. It is fast and not very complicated. Another positive value is the ease of the installation of the cameras and matrices of light points on board. Moreover the system is 
not very sensitive to external factors such as scratches on the ship's sides, rainfall, lack of good lighting and a small mist in comparison to system based on laser measurements.

- Errors in position and angle estimation at the level of $1.5 \%$ of range are acceptable. The results can be improved by taking into account the calibration and optical defects of the registrant. In the area where errors are the biggest is where ships achieve second phase of motion. It may be an area for additional laser rangefinders arranged perpendicular to the side at the bow and stern.

- Future work includes algorithm running in real time and incorporate optical defects such as distortion at short focal lengths to improve its performance.

\section{BIBLIOGRAPHY}

1. Acharya T.: Integrated Color Interpolation and Color Space Conversion Algorithm from 8-bit Bayer Pattern RGB Color Space to 12-bit YCrCb Color Space, Patent No.6,392,69931, May 21, 2002.

2. Amann M.C.: Bosch T., Lescure M., Myllylä R.: Laser ranging: a critical review of usual techniques for distance measurement. Optical Engineering, Vol.40, 10 (2001).

3. Atkinson K. B.: Close range photogrammetry and machine vision, Whittles, 2001.

4. Batlle J., Mouaddib E., Salvi J.: Recent progress in coded structured light as a technique to solve the correspondence problem, Pat. Recog., 31(7), 1998, p. 963-982.
5. Blais F.: Review of 20 years of range sensor development. Journal of Electronic Imaging, Vol. 13(1), 2004, p. 231-240.

6. Bole A., Dineley B., Wall A.: Radar and ARPA manual. Elsevier, Amsterdam-Tokyo, 2006.

7. Gierusz W., Waszkiel A.: Determination of suction forces and moment on parallel manoeuverin vessels for a future control system. Solid State Phenomena, Trans Tech Publications, Switzerland, Vol. 180, 2012, p. 281-187.

8. Samsung S1070 User Manual, www.samsung.com.

9. Tos C.: Non-metric cameras use for cost-estimate survey at civil works, Casopismo Techniczne S, Wyd. Politechniki Krakowskiej, 2-S/2008, p. 283-290.

10.Webster, D.: A pulsed ultrasonic distance measurement system based upon phase digitizing. IEEE Transactions on Instrumentation and Measurement, Vol. 4, 1994, p.578-582.

11.Yilmaz H.M., Yakar M., Gulec S.A., Dulgerler O. N.: Importance of digital close-range photogrammetry in documentation of cultural heritage, Journal of Cultural Heritage, Vol. 8, 2007, p. 428-433.

\section{CONTACT WITH THE AUTHOR}

Anna Waszkiel, M.Sc.

Faculty of Marine Electrical Engineering, Gdynia Maritime University, Morska 81-87 81-225 Gdynia, POLAND e-mail: anwas@am.gdynia.pl 